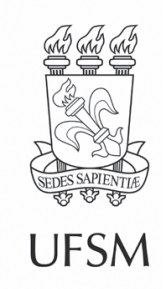

\title{
Artigos
}

\section{Alocação de biomassa e indicadores de crescimento para a avaliação da qualidade de mudas de espécies florestais nativas}

\author{
Biomass allocation and growth indicators for quality evaluation of \\ seedlings of native forest species
}

\author{
Natanielly Rodrigues Avelino ${ }^{\oplus}$, Ana Cristina Schilling ${ }^{\oplus}$, \\ Ândrea Carla Dalmolin" ${ }^{\oplus}$, Martielly Santana dos Santos ${ }^{\circledR}$, Marcelo Schramm Mielke $^{1 \oplus}$ \\ Universidade Estadual de Santa Cruz, Ilhéus, BA, Brasil \\ "Universidade Federal do Sul da Bahia, Ilhéus, BA, Brasil
}

\section{Resumo}

Procedimentos e metodologias que permitam avaliar a qualidade e a uniformidade das mudas são fundamentais para a homogeneização dos lotes em viveiros florestais e o sucesso dos plantios para reflorestamento. Foi conduzido um estudo com o objetivo de avaliar as correlações entre a alocação de biomassa e o índice de qualidade de Dickson (IQD) para mudas de seis espécies arbóreas nativas da Mata Atlântica, bem como selecionar indicadores de qualidade que mais influenciam o IQD e que possam ser utilizados de maneira prática e precisa para a avaliação da qualidade de mudas florestais nativas. Foram utilizadas mudas de seis espécies arbóreas tropicais: Calophyllum brasiliense, Eriotheca macrophylla, Inga laurina, Psidium cattleyanum, Roupala montana e Sloanea obtusifolia. Foram avaliados a massa seca de folhas (MSF), os caules (MSC) e as raízes (MSR), além da altura da parte aérea (H) e diâmetro do coleto (D). A partir dessas variáveis, foram calculadas as razões de massa de raízes (RMR), caules (RMC) e folhas (RMF), além da massa seca total (MST), a massa seca da parte aérea (MSPA), a razão de massa seca da parte aérea por massa seca de raízes (MSPA/MSR), a razão altura da parte aérea por diâmetro do coleto (H/D) e o IQD. Os resultados foram analisados mediante análises de correlação. Com base nas condições em que este estudo foi realizado é possível concluir que: (a) as diferenças entre os valores médios do IQD das espécies estudadas não foram explicadas pelo padrão de alocação de biomassa característico de cada espécie; (b) a MSR, a MST e o D são as melhores variáveis para predizer o comportamento do IQD das mudas das espécies florestais analisadas neste estudo; e (c) pelo fato de ser um método não destrutivo, o D pode ser considerado como o melhor indicador prático para estimar os valores de IQD de lotes de mudas de espécies florestais da Mata Atlântica.

Palavras-chave: Índice de qualidade de Dickson; Mata Atlântica; Viveiros florestais; Restauração florestal 


\begin{abstract}
Procedures and methodologies for evaluating the quality and uniformity of seedlings are essential for homogenizing lots in forest nurseries and successful planting for reforestation. A study was made in order to evaluate the correlations between the allocation of biomass and the Dickson Quality Index (IQD) for seedlings from six native tree species of the Atlantic Forest as well as to select quality indicators that most affect the IQD and which can be used in a practical and precise fashion for the evaluation of native forest seedlings. Seedlings from six tropical forest species were used: Calophyllum brasiliense, Eriotheca macrophylla, Inga laurina, Psidium cattleyanum, Roupala montana and Sloanea obtusifolia. The following characteristics were evaluated: dry leaf (MSF), dry stem (MSC) and dry root (MSR) mass, as well as the aerial part height $(\mathrm{H})$ and the stem diameter $(\mathrm{D})$. Based on these variables, the following calculations were made: ratios of root (RMR), stem (RMC) and leaf (RMF) mass, as well as the total dry mass (MST), that of the aerial part (MSPA), the ratio of the aerial part dry mass to the dry root mass (MSPA/MSR), and that of the aerial part height to the stem diameter (H/D), and the IQD. The results were analyzed via correlation analyses. Based on the conditions of this study it is possible to conclude that: (a) the differences between the average IQD values of the species studied were not explained by the allocation pattern of biomass characterizing each species; (b) the MSR, MST and the D are the best variables for predicting the IQD of the seedlings from the forest species analyzed in this study; and (c) because it is a non-destructive method, D can be considered the best practical indicator for estimating IQD values for Atlantic Forest seedling species lots.
\end{abstract}

Keywords: Dickson quality index; Brazilian Atlantic Forest; Forest nurseries; Forest restoration

\title{
1 INTRODUÇÃO
}

A produção de mudas em viveiros florestais deve ser orientada por critérios técnicos bem elaborados, pois muitos são os fatores que condicionam a qualidade final das mesmas (MATTSSON, 1997). Entre os fatores que condicionam a qualidade final das mudas se destacam a qualidade genética, o estágio de desenvolvimento e a viabilidade das sementes, a escolha do recipiente e do substrato de cultivo, a adubação, as práticas culturais, além do tempo de permanência das mudas no viveiro (CARNEIRO, 1995; HALL, 2003; WILKINSON et al., 2014). Em regiões de clima tropical, devido à grande diversidade de espécies, a avaliação dos lotes é um fator crítico e que requer o aprimoramento constante dos procedimentos e metodologias de avaliação da qualidade das mudas que serão destinadas ao plantio no campo (WILKINSON et al., 2014).

Nos viveiros florestais, a avaliação da qualidade das mudas permitirá a escolha de lotes mais vigorosos e homogêneos para compor o reflorestamento (HAASE; 
DAVIS, 2017). Considerando-se que o crescimento de plantas é definido como sendo o aumento irreversível em matéria e volume de células, tecidos e órgãos, a qualidade das mudas pode ser avaliada por meio de indicadores de crescimento, como a altura da parte aérea $(H)$, o diâmetro do coleto (D) e a biomassa seca de folhas (MSF), caules (MSC), raízes (MSR) e total (MST) (GOMES et al., 2002; HAASE, 2008; NÓBREGA et al., 2008; BINOTTO; LÚCIO; LOPES, 2010; TSAKALDIMI; GANATSAS; JACOBS, 2013). Além desses, vários índices são utilizados para avaliar a qualidade de lotes de mudas florestais, como as razões H/D, massa seca da parte área/MSR (MSPA/MSR) (HAASE, 2008) e o índice de qualidade de Dickson (IQD) (DICKSON; LEAF; HOSNER, 1960).

A razão H/D relaciona dois importantes indicadores de crescimento de mudas em um índice, conhecido também como quociente de robustez (GOMES et al., 2002). A razão H/D é amplamente empregada devido à facilidade de mensurar seus componentes e por se mostrar de alta confiabilidade, expressando o equilíbrio de crescimento da muda (CARNEIRO, 1995). Nessa razão, uma relação menor indica uma muda mais robusta, ou seja, apta a sobreviver e se estabelecer após o plantio em campo, enquanto valores altos indicam mudas relativamente delgadas, mais susceptíveis a danos causados por manuseio, vento, seca e geada (HAASE, 2008).

Outra razão que também mostra ser eficiente para a avaliação da qualidade de lotes de mudas florestais e que está diretamente relacionada com o estabelecimento no campo e na competitividade em condições de estresse ambiente, como o deficit hídrico, é MSPA/MSR (GROSSNICKLE, 2012; GROSSNICKLE; MACDONALD, 2018). Isso porque diferentes fatores afetam o padrão de alocação da biomassa em uma planta, como as características genéticas e a idade, além de fatores do ambiente, como temperatura, radiação luminosa, disponibilidade hídrica e nutricional (CHAMBEL; CLIMENT; ALÍA, 2007), pois a planta destina a biomassa preferencialmente ao órgão que captura o recurso limitante ao crescimento (SHIPLEY; MEZIANE, 2002). A MSR é reconhecida como um dos indicadores mais simples e que melhor determina o estabelecimento das mudas em campo por influenciar diretamente na absorção de água e nutrientes 
(GROSSNICKLE, 2012; SHEN et al., 2019). Assim, plantas com maior MSR serão mais eficazes para o manejo florestal pela capacidade de aclimatação após o transplante. No entanto, a determinação desse indicador pode ter os valores subestimados devido à perda de biomassa radicular no momento da coleta e manuseio.

O IQD tem como base a relação entre diversos indicadores de crescimento já usados para determinar a qualidade das mudas, ou seja, MST, MSPA, MSR, H e D. Por isso, a fórmula do IQD evidencia o equilíbrio entre crescimento e potencial de sobrevivência das plantas no pós-plantio (GOMES, 2001), pois, ao considerar várias características morfológicas, os possíveis erros que poderiam ser encontrados ao usar um ou dois indicadores serão minimizados. Assim, é um ótimo indicador da qualidade das mudas por incluir no seu cálculo a robustez e o equilíbrio da alocação da biomassa (MANÃS; CASTRO; HERAS, 2009). No entanto, o IQD é um índice que fornece valores variáveis que diferem em função da espécie, material genético, condições de cultivo e da idade em que a muda passou pela avaliação (GOMES et al., 2013; GASPARIN et al., 2014). Apesar das grandes variações observadas entre espécies, e mesmo dentro da mesma espécie, o IQD tem sido utilizado como o principal indicador da qualidade de mudas florestais (p. ex. GASPARIN et al., 2014; LEITE et al., 2017; DANTAS et al., 2018; LIMA FILHO et al., 2019; entre outros), uma vez que apresenta altas correlações com a sobrevivência após o plantio no campo (BAYALA et al., 2009; TSAKALDIMI; GANATSAS; JACOBS, 2013). Mesmo com a ampla utilização do IQD, é imprescindível relacioná-lo aos demais indicadores de crescimento, visando à aplicação de procedimentos mais práticos e precisos para a avaliação da qualidade de mudas em viveiros.

A partir disso, o presente estudo tem como objetivos avaliar as correlações entre a alocação de biomassa e o IQD para mudas de seis espécies arbóreas nativas da Mata Atlântica, bem como selecionar indicadores de qualidade que mais influenciam o IQD e que possam ser utilizados de maneira prática e precisa para a avaliação da qualidade de mudas florestais nativas. 


\section{MATERIAL E MÉTODO}

Foram utilizadas mudas de seis espécies arbóreas nativas tropicais da Mata Atlântica do sul da Bahia, a saber: Calophyllum brasiliense Cambess. (Calophyllaceae), Eriotheca macrophylla (K.Schum.) A.Robyns (Malvaceae), Inga laurina (Sw.) Willd. (Fabaceae), Psidium cattleyanum Weinw. (Myrtaceae), Roupala montana Aubl. (Proteaceae) e Sloanea obtusifolia K.Schum. (Elaeocarpaceae). Essas espécies foram escolhidas em função da disponibilidade de sementes e pelo fato de apresentarem padrões de crescimento distintos. As mudas foram produzidas no viveiro do Instituto Floresta Viva, localizado em Serra Grande, distrito de Uruçuca - BA. As mudas analisadas foram produzidas segundo protocolo geral do viveiro. As sementes foram coletadas em fragmentos florestais localizados na região de llhéus e Serra Grande e as semeaduras foram realizadas em canteiro de germinação. Para cada espécie, a semeadura, a repicagem e a amostragem foram realizadas em períodos diferentes (Tabela 1). Após a germinação, as plântulas foram repicadas para tubetes de $115 \mathrm{~cm}^{3}$ preenchidos

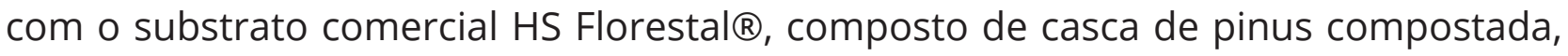
turfa vegetal e vermiculita. A repicagem das plântulas foi feita após a abertura dos cotilédones, priorizando a uniformidade. Os tubetes foram acondicionados em bandejas de capacidade para 108 tubetes e foram distribuídos de forma a adotar uma densidade de $50 \%$ da bandeja. Após a repicagem foram realizadas adubações semanais com nitrogênio (ureia - $60 \mathrm{mg} \cdot \mathrm{dm}^{3}$ ) e potássio (cloreto de potássio - 30 mg.dm-3), o fósforo foi aplicado apenas na primeira adubação (fosfato monoamônico - 150 mg.dm-3). As mudas foram irrigadas todos os dias por meio de microaspersão automática.

Para a amostragem foram avaliadas a massa seca de folhas (MSF), os caules (MSC) e as raízes (MSR), a altura da parte aérea (H) e o diâmetro do coleto (D) (Tabela 1). A H foi avaliada com auxílio de uma régua, do coleto até o ápice e o $D$ foi medido com paquímetro digital, analisado no nível da borda do tubete. Para determinação da 
massa seca, as mudas foram coletadas, lavadas, separadas em folhas, caules e raízes. O material vegetal foi acondicionado separadamente em sacos de papel e colocados para secar em estufa de ventilação forçada a $75^{\circ} \mathrm{C}$ até obter massa constante. A partir desses valores, foram calculadas as seguintes variáveis, com base em Dickson, Leaf e Hosner (1960): massa seca total (MST), em g: MST = MSF + MSC + MSR; massa seca da parte aérea (MSPA), em g: MSPA = MSC + MSF; razão altura da parte aérea por diâmetro do coleto (H/D), em $\mathrm{cm} \mathrm{mm}^{-1}$; razão massa seca da parte aérea por massa seca de raízes (MSPA/MSR), em g; índice de qualidade de Dickson (IQD): IQD = MST / [(H/D) + (MSPA / MSR)]; razão da massa de folhas (RMF): MSF/MST; razão da massa de caules (RMC): MSC/MST; e razão da massa de raízes (RMR): MSR/MST.

A determinação da intensidade da correlação entre as razões de alocação de biomassa e os indicadores de crescimento com o IQD foram analisadas mediante correlação de Pearson. A interpretação das intensidades das correlações foi analisada a partir dos valores dos coeficientes de correlação ( $r$ ), com base na classificação de Hinkle, Wiersma e Jurs (2003). Esses autores consideram correlações: muito forte (0,90 $>r>1,00)$, forte $(0,70>r>0,89)$, moderada $(0,50>r>0,69)$, fraca $(0,30>r>0,49)$ e muito fraca ou ausente $(0,00>r>0,29)$; podendo os valores serem positivos ou negativos. 0 software utilizado para as análises estatísticas foi o R (R CORE TEAM, 2018).

\section{RESULTADOS E DISCUSSÃO}

O tempo de permanência dos lotes de mudas avaliados variou entre 137 dias (Calophyllum brasiliense) e 294 dias após a repicagem (Roupala montana) (Tabela 1). A maior e a menor altura média foram obtidas para os lotes de Psidium cattleyanum e Sloanea obtusifolia, respectivamente, e o maior e menor diâmetro médio foram obtidos para os lotes de Eriotheca macrophylla e Sloanea obtusifolia, respectivamente. Assim como ocorreu para a altura média, os maiores e menores valores médios de massa seca das mudas foram obtidos para os lotes de Psidium cattleyanum e Sloanea 
obtusifolia, respectivamente. Segundo Souza Junior e Brancalion (2016), o tempo de permanência médio no viveiro varia de três a quatro meses (90 a 120 dias), para Calophyllum brasiliense, Inga laurina e Psidium cattleyanum, e quatro a cinco meses (120 e 150 dias) para Roupala montana. Os autores não apresentam informações para Eriotheca macrophylla e Sloanea obtusifolia. Apesar de o tempo de permanência no viveiro ter sido maior do que o recomendado por Souza Júnior e Brancalion (2016), o tamanho das mudas estava dentro da faixa recomendada para Calophyllum brasiliense (20 a 30 cm), Inga laurina (20 a 30 cm) e Psidium cattleyanum (20 a 40 cm). Considerandose uma altura média superior a $20 \mathrm{~cm}$ para a expedição das mudas, apenas os lotes de Roupala montana e Sloanea obtusifolia não tinham atingido altura mínima. Com exceção de Sloanea obtusifolia, todos os outros lotes tinham atingido o diâmetro mínimo de $3 \mathrm{~mm}$, recomendado para a expedição de mudas da maioria das espécies florestais nativas descritas por Souza Júnior e Brancalion (2016).

O maior e o menor valor médio de RMF foram encontrados para Sloanea obtusifolia e Calophyllum brasiliense (Tabela 2). Já para a RMC, a maior e a menor média foram observadas para Psidium cattleyanum e para Roupala montana. Nota-se também que houve uma relação inversa entre a RMF e a RMC. Já para RMR, com exceção de Psidium cattleyanum, as demais espécies apresentaram valores médios em torno de 0,35. O menor valor médio de RMR foi obtido para Psidium cattleyanum. De modo geral, os resultados obtidos para RMF, RMC e RMR indicam que havia uma relação relativamente equitativa na partição de biomassa para folhas, caules e raízes para todas as espécies, indicando também que não havia restrições ao crescimento das raízes em função do tempo de permanência das mudas no viveiro e do tamanho dos tubetes utilizados $\left(115 \mathrm{~cm}^{3}\right)$.

A literatura indica que quanto maior o valor do IQD maior será a sobrevivência após o plantio no campo (BAYALA et al., 2009; BINOTTO; LÚCIO; LOPES, 2010; TSAKALDIMI; GANATSAS; JACOBS, 2013). No entanto, pelo fato de envolver o uso 
de medições destrutivas, como MSR, MSPA e MST, o uso operacional do IQD em viveiros florestais ainda é bastante dificultado para espécies nativas. Além disso, os resultados relatados na literatura para o IQD são muito variáveis. Por exemplo, Leles et al. (2006), pesquisando o tamanho de tubete para a produção de mudas de quatro espécies florestais nativas, reportaram os maiores valores de IQD variando entre 0,78 para Anadenanthera macrocarpa, até 4,05, para Chorisia speciosa. Alonso et al. (2018), testando o uso de biossólidos na composição de substratos para a produção de mudas de Ceiba speciosa (sinonímia Chorisia speciosa), reportaram valores de IQD entre 0,62 e 1,25. Neste estudo, os maiores valores de IQD foram encontrados para Eriotheca macrophylla, seguida de Psidium cattleyanum e Inga laurina (Tabela 2). O baixo valor do IQD encontrado para Sloanea obtusifolia indica que as mudas dessa espécie ainda não estavam aptas ao plantio. Além disso, nesse caso específico, o tempo de permanência das mudas de Sloanea obtusifolia no viveiro (183 dias, ver Tabela 1) foi menor do que o necessário para a boa formação das mudas dessa espécie, o que pode ser relacionado ao crescimento lento da espécie, que pertence ao grupo das espécies não pioneiras (BARBOSA et al., 2017).

Tabela 1 - Valores médios dos indicadores de crescimento e qualidade para mudas de seis espécies arbóreas tropicais com seus respectivos tempos de cultivo

\begin{tabular}{lccccccccc}
\hline \multicolumn{1}{c}{ Espécies } & DAS & DAR & H & D & MSF & MSC & MSR & MST & N \\
\hline Calophyllum brasiliense & 173 & 137 & 25,87 & 4,23 & 0,69 & 0,77 & 0,82 & 2,28 & 48 \\
Eriotheca macrophylla & 218 & 210 & 18,84 & 8,68 & 1,61 & 1,67 & 1,74 & 5,02 & 48 \\
Inga laurina & 189 & 180 & 27,82 & 5,72 & 1,45 & 1,40 & 1,63 & 4,48 & 36 \\
Psidium cattleyanum & 333 & 237 & 37,87 & 5,33 & 2,77 & 2,65 & 2,14 & 7,57 & 47 \\
Roupala montana & 334 & 294 & 14,75 & 4,16 & 1,15 & 0,70 & 0,96 & 2,81 & 48 \\
Sloanea obtusifolia & 183 & 176 & 11,52 & 2,89 & 0,26 & 0,18 & 0,23 & 0,67 & 36 \\
\hline
\end{tabular}

Fonte: Autores (2020)

Em que: Dias após a semeadura (DAS), dias após a repicagem (DAR), altura (H, $\mathrm{cm})$, diâmetro do coleto (D, mm), massa seca de folhas (MSF, g), massa seca de caules (MSC, g), massa seca de raízes (MSR, g), massa seca total (MST, g) e número de plantas (N). 
Correlações negativas muito fortes e fortes encontradas para as correlações entre RMF e RMC ou RMR (Tabela 3) sugerem a ocorrência de distribuição preferencial da biomassa vegetal entre os órgãos; ou seja, enquanto a biomassa alocada para folhas aumentou, diminuiu a alocação de biomassa para caules e raízes. Para Roupala montana, a correlação positiva forte entre RMR e RMC indica que, para essa espécie, parece ocorrer uma forte competição entre a biomassa alocada para folhas em relação à biomassa alocada para caules e raízes. Quando todas as espécies foram analisadas em conjunto, nota-se que as correlações entre RMF e RMC ou RMR foram fortes e negativas, e a correlação entre RMC e RMR foi fraca ou ausente. A alocação de biomassa é uma estratégia de grande importância para a adaptação e aclimatação das espécies vegetais aos diferentes ambientes de crescimento (MARKESTEIJN; POORTER, 2009). Os padrões de alocação de biomassa variam em função da espécie, da ontogenia e do ambiente de crescimento (POORTER et al., 2012). Considerando-se que todas as plantas utilizadas neste estudo se encontravam no mesmo estágio ontogenético, e foram cultivadas nas mesmas condições de clima, substrato e embalagem, as diferenças encontradas para as correlações entre RMF, RMC e RMR indicam uma grande variação nos padrões de alocação de biomassa entre as espécies analisadas.

Tabela 2 - Valores médios das razões de massa de folhas (RMF), de caules (RMC) e raízes (RMR), da razão altura e diâmetro do coleto (H/D), da razão massa seca da parte aérea e raízes (MSPA/MSR) e do índice de qualidade de Dickson (IQD) para lotes de mudas de seis espécies arbóreas tropicais

\begin{tabular}{lcccccc}
\hline \multicolumn{1}{c}{ Espécies } & RMF & RMC & RMR & H/D & MSPA/MSR & IQD \\
\hline Calophyllum brasiliense & 0,30 & 0,34 & 0,36 & 6,22 & 1,82 & 0,29 \\
Eriotheca macrophylla & 0,33 & 0,33 & 0,34 & 2,20 & 2,07 & 1,26 \\
Inga laurina & 0,33 & 0,31 & 0,36 & 4,92 & 1,79 & 0,69 \\
Psidium cattleyanum & 0,35 & 0,36 & 0,29 & 7,16 & 2,58 & 0,80 \\
Roupala montana & 0,37 & 0,27 & 0,37 & 3,59 & 1,94 & 0,51 \\
Sloanea obtusifolia & 0,38 & 0,27 & 0,35 & 4,02 & 1,92 & 0,12 \\
\hline
\end{tabular}

Fonte: Autores (2020)

Em que: *Símbolos e número de plantas por espécie (ver Tabela 1). 
Tabela 3 - Coeficiente de correlação de Pearson (r) entre variáveis de alocação de biomassa para mudas de seis espécies arbóreas tropicais

\begin{tabular}{lccccccc}
\hline & CB & EM & IL & PC & RM & SO & Todas \\
\hline RMF x RMC & $-0,61$ & $-\mathbf{0 , 7 0}$ & $-0,40$ & $-\mathbf{0 , 8 9}$ & $-\mathbf{0 , 9 2}$ & $-0,48$ & $-\mathbf{0 , 7 1}$ \\
RMF x RMR & $-\mathbf{0 , 7 2}$ & $-\mathbf{0 , 7 7}$ & $-0,55$ & $-0,64$ & $-\mathbf{0 , 9 7}$ & $\mathbf{- 0 , 8 3}$ & $\mathbf{- 0 , 7 5}$ \\
RMC $\times$ RMR & $-0,10$ & 0,08 & $-0,54$ & 0,22 & $\mathbf{0 , 8 1}$ & $-0,08$ & 0,08 \\
\hline
\end{tabular}

Fonte: Autores (2020)

Em que: razão da massa de folhas (RMF), razão da massa de caules (RMC), razão da massa de raízes (RMR). *Símbolos e número de plantas por espécie, favor ver Tabela 1. Apenas correlações fortes ou muito fortes (HINKLE; WIERSMA; JURS, 2003) estão destacadas em negrito.

Não foram observadas correlações muito fortes entre as razões de alocação de biomassa e o IQD, com exceção de Eriotheca macrophylla, que teve uma correlação forte entre RMR e IQD (Tabela 4). Esses resultados indicam que os padrões de alocação de biomassa das espécies estudadas apresentam pouca interferência nos valores de IQD. O caso particular de Eriotheca macrophylla indica que quanto maior a RMR maior será o IQD. Segundo Gomes e Paiva (2011), a produção de raízes tem muita influência na sobrevivência e no crescimento inicial das mudas após o plantio. Ou seja, plantas com raízes mais desenvolvidas terão maior capacidade de aclimatação às novas condições encontradas após o plantio no campo.

Todas as correlações entre MST, MSF, MSC, MSR, MSPA e o IQD foram positivas (Tabela 5). No entanto, apenas as correlações entre MSR e IQD, e MST e IQD foram muito fortes ou fortes para todas as espécies do estudo. Esses resultados indicam claramente uma alta influência de MST e MSR nos valores do IQD. Resultados semelhantes foram encontrados por Binotto, Lucio e Lopes (2010) que obtiveram correlações positivas muito fortes entre massa seca de raízes, massa seca total e o IQD para Eucalyptus grandis e Pinus elliottii. Para Eloy et al. (2013), a obtenção da massa seca das mudas é um dos melhores métodos para determinar sua qualidade, sendo que seu valor indica a rusticidade da muda.

Diversos autores consideram o índice MSPA/MSR eficiente para avaliar a 
qualidade das mudas e ainda sugerem que o valor dessa relação seja igual a 2,0 (GOMES; PAIVA, 2011). No presente estudo, com exceção de Psidium cattleyanum, todas as demais espécies apresentaram valores de MSPA/MSR inferiores ou muito próximos de 2,0 (Tabela 2). Apesar de ser um índice indicado para a avaliação da qualidade de mudas (GOMES; PAIVA, 2011), valores muito altos da razão MSPA/MSR podem resultar em um desbalanço entre a absorção de água pelas raízes e perda de água pela transpiração (GROSSNICKLE, 2012), indicando menor qualidade das mudas. Os resultados obtidos no presente estudo para as correlações entre a MSPA/MSR e o IQD (Tabela 5) indicam que a alocação preferencial de biomassa para a parte aérea em relação à alocação de biomassa para as raízes possui influência mínima, ou até mesmo negativa, nos valores do IQD. As correlações negativas entre MSPA/MSR e o IQD podem ser explicadas pelo fato de que este índice se encontra denominador da fórmula do IQD. Assim, quanto maiores forem os valores de MSPA/MSR, menores serão os valores do IQD. Apesar de terem sido encontradas correlações positivas fortes e muito fortes entre a MSPA e o IQD para quatro das seis espécies utilizadas no presente estudo, os resultados obtidos para as correlações entre a razão MSPA/MSR indicam que a MSR está mais correlacionada com o IQD do que a MSPA.

Tabela 4 - Coeficientes de correlação de Pearson ( $r$ ) entre variáveis de alocação de biomassa e o índice de qualidade de Dickson (IQD) para mudas de seis espécies arbóreas tropicais

\begin{tabular}{lccccccc}
\hline & CB & EM & IL & PC & RM & SO & Todas \\
\hline RMF $\times$ IQD & $-0,32$ & $-0,55$ & $-0,39$ & 0,31 & 0,22 & 0,10 & $-0,09$ \\
RMC $\times$ IQD & 0,16 & 0,02 & $-0,12$ & $-0,45$ & $-0,29$ & $-0,25$ & 0,13 \\
RMR $\times$ IQD & 0,27 & $\mathbf{0 , 7 4}$ & 0,46 & 0,09 & $-0,17$ & 0,04 & 0,00 \\
\hline
\end{tabular}

Fonte: Autores (2020)

Em que: razão da massa de folhas (RMF), razão da massa de caules (RMC), razão da massa de raízes (RMR). * Símbolos e número de plantas por espécie, favor ver Tabela 1. Apenas correlações fortes ou muito fortes (HINKLE; WIERSMA; JURS, 2003) estão destacadas em negrito. 
Tabela 5 - Coeficientes de correlação de Pearson ( $r$ ) entre os indicadores de crescimento e o índice de qualidade de Dickson (IQD) para mudas de seis espécies arbóreas tropicais

\begin{tabular}{lccccccc}
\hline & CB & EM & IL & PC & RM & SO & Todas \\
\hline MST x IQD & $\mathbf{0 , 8 4}$ & $\mathbf{0 , 9 4}$ & $\mathbf{0 , 8 8}$ & $\mathbf{0 , 8 2}$ & $\mathbf{0 , 8 1}$ & $\mathbf{0 , 9 6}$ & $\mathbf{0 , 7 6}$ \\
MSF $\times$ IQD & 0,43 & 0,64 & 0,59 & 0,65 & 0,60 & $\mathbf{0 , 8 5}$ & 0,60 \\
MSC $\times$ IQD & $\mathbf{0 , 7 0}$ & $\mathbf{0 , 8 9}$ & 0,69 & 0,61 & 0,68 & $\mathbf{0 , 8 5}$ & 0,70 \\
MSR x IQD & $\mathbf{0 , 9 3}$ & $\mathbf{0 , 9 9}$ & $\mathbf{0 , 8 8}$ & $\mathbf{0 , 8 2}$ & $\mathbf{0 , 8 8}$ & $\mathbf{0 , 8 6}$ & $\mathbf{0 , 8 6}$ \\
MSPA x IQD & 0,67 & $\mathbf{0 , 8 5}$ & $\mathbf{0 , 7 3}$ & $\mathbf{0 , 7 5}$ & 0,68 & $\mathbf{0 , 8 9}$ & 0,68 \\
MSPA/MSR x IQD & $-0,28$ & $-0,67$ & $-0,47$ & $-0,10$ & 0,15 & $-0,13$ & $-0,02$ \\
H x IQD & $-0,13$ & 0,36 & $-0,43$ & $-0,23$ & 0,07 & 0,18 & 0,18 \\
D x IQD & $\mathbf{0 , 8 1}$ & $\mathbf{0 , 8 7}$ & $\mathbf{0 , 7 8}$ & 0,66 & $\mathbf{0 , 7 9}$ & $\mathbf{0 , 7 6}$ & $\mathbf{0 , 8 9}$ \\
H/D $\times$ IQD & $-\mathbf{0 , 7 9}$ & $-0,53$ & $-\mathbf{0 , 8 3}$ & $-0,50$ & $-0,39$ & $\mathbf{- 0 , 8 2}$ & $-0,36$ \\
\hline
\end{tabular}

Fonte: Autores (2020)

Em que: massa seca total (MST), massa seca de folhas (MSF), massa seca de caules (MSC), massa seca de raízes (MSR), massa seca da parte aérea (MSPA), razão massa seca da parte aérea e massa seca de raízes (MSPA/MSR), altura $(H)$, diâmetro do coleto (D), razão altura e diâmetro do coleto (H/D). *Símbolos e número de plantas por espécie, favor ver Tabela 1. Apenas correlações fortes ou muito fortes (HINKLE; WIERSMA; JURS, 2003) estão destacadas em negrito.

Correlações fracas ou muito fracas entre H e IQD indicam que a altura da parte aérea pouco contribuiu no valor do IQD das espécies analisadas (Tabela 5). Correlações negativas entre a razão H/D foram encontradas para todas as seis espécies utilizadas no estudo. Assim como ocorreu para a razão MSPA/MSR, as correlações negativas entre H/D e o IQD podem ser explicadas pelo fato de que este índice se encontra denominador da fórmula do IQD. Quanto maiores forem os valores de H/D, menores serão os valores do IQD. É importante salientar que, embora os valores de MSPA/MSR tenham tido uma variação relativamente pequena entre as espécies analisadas (Tabela 2), foi observada uma grande variação para a razão H/D. Por exemplo, o valor médio de H/D foi cerca de três vezes maior para Psidium cattleyanum do que para Eriotheca macrophylla. Apesar de a correlação entre H/D e o IQD ter sido fraca quando todas as espécies foram consideradas em conjunto, correlações negativas e fortes foram observadas para três das seis espécies analisadas. Além disso, mudas altas e com 
diâmetro médio do coleto pequeno podem ter maiores chances de tombamento após o plantio no campo (GASPARIN et al., 2014). Lima Filho et al. (2019) observaram que a análise conjunta dos valores de H e D permitiu uma melhor avaliação da capacidade de sobrevivência e o crescimento de mudas de Ceiba speciosa após o plantio no campo. Assim, sugere-se que a $\mathrm{H}$ não seja utilizada de modo isolado para determinar a qualidade das mudas.

Correlações positivas e fortes ou muito fortes entre o D e o IQD foram observadas para cinco das seis espécies, e quando todas as espécies foram consideradas em conjunto (Tabela 5). Da mesma forma como ocorreu para MSR, as correlações positivas verificadas para o D são decorrentes da fórmula do IQD. No entanto, as correlações fortes ou muito fortes indicam que o diâmetro do coleto contribuiu fortemente para o aumento do IQD. De fato, o D já vem sendo considerado como um ótimo indicador do desempenho das mudas após o plantio em campo (BAYALA et al., 2009; TSAKALDIMI; GANATSAS; JACOBS, 2013). A importância de um caule robusto, com maior diâmetro, pode ser relacionada às pequenas variações que naturalmente ocorrem ao longo do dia, dependendo da espécie, demanda evaporativa do ar e da disponibilidade de água no solo, atuando assim como um tampão hídrico em condições de estresse ambiente (SWAEF et al., 2015). Essa oscilação diária no diâmetro do caule, por estar associada ao status hídrico da planta influenciando também a MSR e, consequentemente, O IQD. Tais fatos justificam fisiologicamente as correlações positivas e fortes de D e MSR com o IQD, e as indicações dessas variáveis como os melhores índices para a avaliação da qualidade de mudas.

Apesar das altas correlações entre a MST e MSR com o IQD, a determinação da massa seca é pouco indicada para a avaliação da qualidade das mudas em viveiros florestais, pois se trata de um método destrutivo de avaliação e que necessita de certa infraestrutura, como estufas e balanças (TSAKALDIMI; GANATSAS; JACOBS, 2013). Desse modo, o D destaca-se por ser um método de avaliação não destrutivo e de fácil 
mensuração, além de ser um método já empregado nas avaliações de qualidade de mudas (BAYALA et al., 2009; BINOTTO; LÚCIO; LOPES, 2010; TSAKALDIMI; GANATSAS; JACOBS, 2013). Por outro lado, o D é geralmente obtido por meio de paquímetros analógicos ou digitais. Paquímetros analógicos podem ser confeccionados em plástico ou metal e são equipamentos bastante robustos para o uso em viveiros. No entanto, esses equipamentos são de difícil leitura e exigem muita atenção para o seu uso correto, podendo ocasionar fadiga do operador e erros de medição quando os lotes de mudas forem muito grandes. O paquímetro digital é um aparelho de fácil manuseio e é facilmente encontrado para venda em lojas especializadas em ferramentas de precisão. No entanto, seu uso em viveiros florestais apresenta restrições, pois é um aparelho muito delicado para se manusear, o que eleva os riscos de danificar o equipamento. Diante dessa limitação, propõe-se a elaboração de um medidor de diâmetro mais resistente aos processos operacionais que envolvem a produção de mudas. Sugere-se que o medidor seja construído com materiais mais resistentes como placas de inox ou acrílico, por exemplo, e que tenha uma série de pequenas ranhuras com diâmetros conhecidos. Nesse caso, as ranhuras poderiam ser padronizadas dentro de faixas de valores de diâmetros predefinidos para mudas de espécies arbóreas nativas.

\section{CONCLUSÕES}

Com base nas condições em que este estudo foi realizado, pode-se concluir que: (a) as diferenças entre os valores médios do IQD das espécies estudadas não foram explicadas pelo padrão de alocação de biomassa característico de cada espécie; (b) a MSR, a MST e o D são as melhores variáveis para predizer o comportamento do IQD das mudas das espécies florestais analisadas neste estudo; e (c) pelo fato de ser um método não destrutivo, o D pode ser considerado como o melhor indicador prático para estimar os valores de IQD de lotes de mudas de espécies florestais da Mata Atlântica. 


\section{AGRADECIMENTOS}

Os autores agradecem ao Eng. Agrônomo e Mestre em Produção Vegetal Murilo F. C. de Jesus e aos técnicos do Viveiro Comunitário Floresta Viva Gerson J. Sales Neto, Nilson A. dos Santos e Rones F. Souza, pelo auxílio na coleta dos dados, e a Professora Angela Van Erven Cabala pela revisão da escrita em inglês. Natanielly R. Avelino agradece à Fundação de Apoio à Pesquisa do Estado da Bahia (Fapesb) pela concessão da bolsa de mestrado (BOL0023/2018). O presente trabalho foi realizado com apoio do Conselho Nacional de Desenvolvimento Tecnológico (CNPq, Processo 561933/2010-3) e da Coordenação de Aperfeiçoamento de Pessoal de Nível Superior - Brasil (CAPES) - Código de Financiamento 001. Ândrea C. Dalmolin (307604/2020-9) e Marcelo S. Mielke (305477/2018-8) agradecem ao CNPq pelas bolsas de produtividade em pesquisa.

\section{REFERÊNCIAS}

ALONSO, J. M. et al. Biosolids as substrate for the production of Ceiba speciosa seedlings. Cerne, Lavras, v. 24, n. 4, p. 420-429, 2018.

BARBOSA, L. M. et al. Lista de espécies indicadas para restauração ecológica para diversas regiões do estado de São Paulo. São Paulo: Instituto de Botânica, 2017. 344 p.

BAYALA, J. et al. Predicting field performance of five irrigated tree species using seedling quality assessment in Burkina Faso, West Africa. New Forests, Basileia, v. 38, p. 309-322, 2009.

BINOTTO, A. F.; LÚCIO, A. D. C.; LOPES, S. J. Correlations between growth variables and the Dickson quality index in forest seedlings. Cerne, Lavras, v. 16, p. 457-464, 2010.

CARNEIRO, J. G. A. Produção e controle de qualidade de mudas florestais. Curitiba: UFPR; FUPEF; UNEF, 1995. 451 p.

CHAMBEL, M. R.; CLIMENT, J.; ALÍA, R. Divergence among species and populations of Mediterranean pines in biomass allocation of seedlings grown under two water regimes. Annals of Forest Science, Paris, v. 64, p. 87-97, 2007.

DANTAS, R. P. et al. Qualidade de mudas de Tabebuia aurea (Manso) Benth. \& Hook. em dois ambientes e diferentes níveis de fertirrigação. Ciência Florestal, Santa Maria, v. 28, n. 3, p. 1253-1262, 2018.

DICKSON, A.; LEAF, A.; HOSNER, J. Quality appraisal of white spruce and white pine seedling stock in nurseries. Forestry Chronicle, Mattawa, v. 36, p. 3-10, 1960. 
ELOY, E. et al. Avaliação da qualidade de mudas de Eucalyptus grandis utilizando parâmetros morfológicos florestal. Floresta, Curitiba, v. 43, n. 3, p. 373-384, 2013.

GASPARIN, E. et al. Influência do substrato e do volume de recipiente na qualidade das mudas de Cabralea canjerana (Vell.) Mart. em viveiro e no campo. Ciência Florestal, Santa Maria, v. 24, n. 3, p. 553-563, 2014.

GOMES, D. R. et al. Lodo de esgoto como substrato para a produção de mudas de Tectona grandis L. Cerne, Lavras, v. 19, n. 1, p. 123-131, 2013.

GOMES, J. M. et al. Parâmetros morfológicos na avaliação da qualidade de mudas de Eucalyptus grandis. Revista Árvore, Viçosa, MG, v. 26, n. 6, p. 655-664, 2002.

GOMES, J. M. Parâmetros morfológicos na avaliação da qualidade de mudas de Eucalyptus grandis, produzidas em diferentes tamanhos de tubete e de dosagens de N-P-K. 2001. Tese (Doutorado) - Universidade Federal de Viçosa, Viçosa, MG, 2001.

GOMES, J. M.; PAIVA, H. N. Viveiros florestais. Viçosa, MG: Editora UFV, 2011. 116 p. (Série Didática).

GROSSNICKLE, S. C. Why seedlings survive: influence of plant attributes. New Forests, Basileia, v. 43, n. 5/6, p. 711-738, 2012.

GROSSNICKLE, S. C.; MACDONALD, J. E. Why seedlings grow: influence of plant attributes. New forests, Basileia, v. 49, n. 1, p. 1-34, 2018.

HAASE, D. L. Understanding forest seedling quality: measurements and interpretation. Tree Planters Notes, Athens, v. 52, n. 2, p. 24-30, 2008.

HAASE, D. L.; DAVIS, A. S. Developing and supporting quality nursery facilities and staff are necessary to meet global forest and landscape restoration needs. Reforesta, Belgrado, v. 4, p. 69-93, 2017.

HALL, C. H. Manual on nursery practices. Kingston: Forestry Department, Ministry of Agriculture, 2003. 77 p.

HINKLE, A.; WIERSMA, W.; JURS, S. G. Statistics applied to behavioral sciences. 5th ed. Boston: Houghton Mifflin, 2003.

LEITE, T. S. et al. Produção de mudas de Enterolobium contortisiliquum e partição de assimilados em resposta à adubação fosfatada e inoculação com fungos micorrízicos. Ciência Florestal, Santa Maria, v. 27, n. 4, p. 1157-1166, 2017.

LELES, P. S. S. et al. Qualidade de mudas de quatro espécies florestais produzidas em diferentes tubetes. Floresta e Ambiente, Rio de Janeiro, v. 13, n. 1, p. 69-78, 2006.

LIMA FILHO, P. et al. Produção de mudas de Ceiba speciosa em diferentes volumes de tubetes utilizando o biossólido como substrato. Ciência Florestal, Santa Maria, v. 29, n. 1, p. 27-39, 2019.

MANÃS, P.; CASTRO, E.; HERAS, J. Quality of maritime pine (Pinus pinaster Ait.) seedlings using waste materials as nursery growing media. New Forests, Basileia, v. 37, p. 295-311, 2009. 
MARKESTEIJN, L.; POORTER, L. Seedling root morphology and biomass allocation of 62 tropical tree species in relation to drought- and shade-tolerance. Journal of Ecology, London, v. 97, p. 311-325, 2009.

MATTSSON, A. Predicting field performance using seedling quality assessment. New Forests, Basileia, v. 13, p. 227-252, 1997.

NÓBREGA, R. S. A. et al. Parâmetros morfológicos de mudas de Sesbania virgata (Caz.) Pers e de Anadenanthera peregrina (L.) cultivadas em substrato fertilizado com composto de lixo urbano. Revista Árvore, Viçosa, MG, v. 32, n. 3, p. 597-607, 2008.

POORTER, H. et al. Biomass allocation to leaves, stems and roots: meta-analyses of interspecific variation and environmental control. New Phytologist, Hoboken, v. 193, p. 30-50, 2012.

R CORE TEAM. R: a language and environment for statistical computing. Vienna: R Foundation for Statistical Computing, 2018. Disponível em: https://www.R-project.org/. Acesso em: 15 mar. 2021.

SHEN, Y. et al. Coordination of leaf, stem and root traits in determining seedling mortality in a subtropical forest. Forest Ecology and Management, Amsterdam, v. 446, p. 285-292, 2019.

SHIPLEY, B.; MEZIANE, D. The balance-growth hypothesis and the allometry of leaf and roots biomass allocation. Functional Ecology, Londres, v. 16, p. 326-31, 2002.

SOUZA JÚNIOR, C. N.; BRANCALION, P. H. S. Sementes e mudas: guia para propagação de árvores brasileiras. São Paulo: Oficina de Textos, 2016. 463 p.

SWAEF, T. et al. Stem diameter variations as a versatile research tool in ecophysiology. Tree Physiology, Oxford, v. 35, p. 1047-1061, 2015.

TSAKALDIMI, M.; GANATSAS, P.; JACOBS, D. F. Prediction of planted seedling survival of five Mediterranean species based on initial seedling morphology. New Forests, Basileia, v. 44, p. 327-339, 2013.

WILKINSON, K. M. et al. Tropical nursery manual: a guide to starting and operating a nursery for native and traditional plants. Washington, DC: Department of Agriculture, 2014. 378 p. (Forest Service Agriculture Handbook, 732).

\section{Contribuição de Autoria}

\section{1 - Natanielly Rodrigues Avelino}

Engenheira Agrônoma, Ma.

https://orcid.org/0000-0003-2493-728X•nataniellyavelino@hotmail.com

Contribuição: Conceituação, Análise Formal, Investigação, Metodologia, Escrita primeira redação, Escrita - revisão e ediç̧ão 


\section{2 - Ana Cristina Schilling}

Engenheira Florestal, Dra., Professora

https://orcid.org/0000-0002-9419-9051•ana.schilling@gmail.com

Contribuição: Conceituação, Metodologia, Visualização de dados, Escrita - primeira redação

\section{3 - Ândrea Carla Dalmolin}

Bióloga, Dra., Professora

https://orcid.org/0000-0002-4372-0492 • andreacarlad@gmail.com

Contribuição: Conceituação, Metodologia, Escrita - primeira redação, Escrita - revisão e edição

\section{4 - Martielly Santana dos Santos}

Bióloga, Dra., Professora

https://orcid.org/0000-0002-0909-2664•martiellysantana@gmail.com

Contribuição: Conceituação, Investigação, Metodologia, Administração do projeto, Recursos, Software, Supervisão, Escrita - primeira redação, Escrita - revisão e edição

\section{5 - Marcelo Schramm Mielke}

Engenheiro Agrônomo, Dr. Professor

https://orcid.org/0000-0001-6930-2902•msmielke@uesc.br

Contribuição: Conceituação, Obtenção de financiamento, Administração do projeto, Supervisão, Escrita - primeira redação, Escrita - revisão e edição

\section{Como citar este artigo}

Avelino, N. R.; Schilling, A. C.; Dalmolin, Â. C.; Santos, M. S.; Mielke, M. S. Alocação de biomassa e indicadores de crescimento para a avaliação da qualidade de mudas de espécies florestais nativas. Ciência Florestal, Santa Maria, v. 31, n. 4, p. 1733-1750, 2021. DOI 10.5902/1980509843229. Disponível em: https://doi.org/10.5902/1980509843229. 